${ }^{6}$ Wilhelm Magnus \& Fritz Oberhettinger, Formeln und Sätze für die Speziellen Funktionen der Mathematischen Physik, Springer, Berlin, 1948.

7 F. JaHnke \& F. EMDE, Tables of Functions, Dover, New York, 1945.

${ }^{8}$ NBSCL, Tables of Bessel Functions $Y_{0}(z)$ and $Y_{1}(z)$ for Complex Arguments, Columbia Press, New York, 1950.

9 NBS Applied Mathematics Series No. 23, Tables of the Normal Probability Function, U. S. Govt. Printing Office, Washington, 1953.

${ }^{10} \mathrm{~L}$. SchwaRz, Untersuchung einiger mit den Zylinderfunktionen nullter Ordnung verwandter Funktionen. Luftfahrtforschung, v. 20,1944, p. 341-372.

$11 \mathrm{G}$. N. Watson, A Treatise on the Theory of Bessel Functions, Macmillan, New York, 1948.

12 For those values of $z$ and $n$ which are of interest here, the difference between $\log _{10} J_{n}(z)$ and $\log _{10} I_{n}(z)$ is approximately equal to $z^{2} \log _{10} e / 2(n+1)$.

\title{
Trapezoidal Methods of Approximating Solutions of Differential Equations
}

Introduction. Seemingly by historical accident the points of view usually adopted for stepwise methods of numerical solution of differential equations largely emphasize the discrete values found rather than the functions pieced together by the method over various intervals of advance. The authors, collaborating as a professor and student team, have found that the straightforward selection of functions to approximate solution functions offers many advantages conceptually and makes it possible to see much-used methods in a new light. We present here a few of the simpler results.

The purpose of this paper is to show that, by considering the method called the trapezoidal method (cf. MILnE [1] p. 24) as a parabolic or quadratic function method, not only does one obtain a satisfying geometrical picture of an approximation curve in the usual case, but that two new trapezoidal or parabolic methods are suggested. Of the two new methods, one is based on a Gauss integration formula. Thus the new approach makes it possible to use the Gauss integration formulas. We believe that the two methods are new in their application although they are old in their use of polynomials. We have found in certain applications that both the new formulas have points of preference in some cases over the simple trapezoidal method.

Quadratic Functions and Trapezoids. Let $y^{\prime}=f(x, y)$ and let it be supposed that $\left(x_{0}, y_{0}\right)$ is either an initial point or that we are now to treat it as such and that we wish to establish a value approximating the solution for $x=x_{1}$. Then, using Milne's terminology, we write

$$
y_{1}-y_{0}=\frac{h}{2}\left(y_{1}^{\prime}+y_{0}^{\prime}\right), \quad h=x_{1}-x_{0} .
$$

From (1) it is clear that, if the solution were known, then (1) is a trapezoidal rule for establishing the integral from the integrand values at the ends of the interval. In case of a differential equation in which $f(x, y)$ involves $y$, however, we do not know $y_{1}$ and if we agree to use $y_{1}{ }^{\prime}=f\left(x_{1}, y_{1}\right)$ we do not know $y_{1}{ }^{\prime}$. Hence, (1) may be considered as an implicit equation for $y_{1}$ which, with adequate restrictions on $h$, yields directly an iteration procedure converging to a unique value $y_{1}$.

Now it is clear that in general $y_{1}$, as finally obtained, is not exactly the value 
of the desired solution function at $x=x_{1}$ and hence calling (1) a trapezoidal method is a concession to form rather than an exact interpretation of the trapezoidal integration of a known function. To understand what is going on, we choose a function $u(x)=a_{0}+a_{1}\left(x-x_{0}\right)+a_{2}\left(x-x_{0}\right)^{2}$ which we want to use as an approximation to the solution from $x_{0}$ to $x_{1}$. We require that

$$
\left\{\begin{aligned}
u\left(x_{0}\right) & =y_{0} \\
u^{\prime}\left(x_{0}\right) & =y_{0}^{\prime}=f\left(x_{0}, y_{0}\right), \\
u^{\prime}\left(x_{1}\right) & =f\left(x_{1}, u_{1}\right)
\end{aligned}\right.
$$

where we write $u_{1}=u\left(x_{1}\right)$. Geometrically, a quadratic function $u(x)$ which satisfies these conditions gives a parabola through $\left(x_{0}, y_{0}\right)$ which has the "direction" $f\left(x_{0}, y_{0}\right)$ at $\left(x_{0}, y_{0}\right)$ and which has the field direction $f\left(x_{1}, u\left(x_{1}\right)\right)$ at $x=x_{1}$. This function is interpreted as an approximation to the solution function for $x_{0} \leq x \leq x_{1}$. The first two equations in (2) give $a_{0}=y_{0}$ and $a_{1}=y_{0}{ }^{\prime}$. Using these in the last equation (2) we have an equation for $a_{2}$ as follows:

$$
2 a_{2} h=f\left(x_{1}, y_{0}+y_{0}^{\prime} h+a_{2} h^{2}\right)-y_{0}^{\prime} .
$$

In general this equation cannot be explicitly solved for $a_{2}$. However, if we assume that a solution $a_{2}$ of (3) is determined and write $f\left(x_{1}, y_{0}+y_{0}^{\prime} h+a_{2} h^{2}\right)$ $=f\left(x_{1}, u_{1}\right)=y_{1}{ }^{\prime}$ then we have on substitution from (3) for $a_{2}$

$$
u(x)=y_{0}+y_{0}{ }^{\prime}\left(x-x_{0}\right)+\frac{y_{1}{ }^{\prime}-y_{0}{ }^{\prime}}{2 h}\left(x-x_{0}\right)^{2} .
$$

With $x=x_{1}, u\left(x_{1}\right)=y_{1}$ we have exactly the "trapezoidal" method (1). We do not recommend using (3) to find $a_{2}$; on the contrary, it is usually greatly preferable to use the iteration suggested by (1). However, in (4) we have, with the solution for $y_{1}$ and $y_{1}{ }^{\prime}$, a quadratic function which may be considered as the approximating function from $x_{0}$ to $x_{1}$. We have never seen this function mentioned in papers or treatises on numerical methods. Continuation of the solution method through several steps gives this picture: the approximating curve consists of continuously and smoothly joined parabolic arcs. Thus the method is theoretically symmetrical or reversible, a terminology we have never seen applied elsewhere. In some cases reverse integration of a differential equation offers some hope of estimating effects of roundoff errors, especially with theoretically reversible or symmetrical methods.

While it is not necessary to interpret the trapezoidal method by a quadratic approximating function, it is simplest to do so. Now, as an integration formula (1) holds exactly for every quadratic function $y(x)$. The error term has the form $-\frac{h^{3}}{12} y^{\prime \prime \prime}$ for continuously thrice differentiable functions $y(x)$. We consider the quadratic approximation function

$$
u(x)=y_{0}+y_{0}^{\prime}\left(x-x_{0}\right)+a_{2}\left(x-x_{0}\right)^{2},
$$

and we ask in addition that

$$
u_{p}^{\prime}=f\left(x_{p}, u_{p}\right)
$$

where

$$
x_{p}=x_{0}+p h, \quad u_{p}=u\left(x_{p}\right), \quad \text { and } \quad u_{p}^{\prime}=u^{\prime}\left(x_{p}\right) .
$$


We consider the number $p$ as a characteristic value to be chosen to our advantage later. Assuming a solution to (6) for $u_{p}=y_{p}$ and letting $u_{p}{ }^{\prime}=y_{p}{ }^{\prime}$, we then have that (5) and (6) give

$$
u(x)=y_{0}+y_{0}^{\prime}\left(x-x_{0}\right)+\frac{y_{p}{ }^{\prime}-y_{0}{ }^{\prime}}{2 p h}\left(x-x_{0}\right)^{2} .
$$

Now letting $u\left(x_{1}\right)=y_{1}$ we have

$$
y_{1}=y_{0}+\frac{h}{2 p}\left[y_{p}{ }^{\prime}+(2 p-1) y_{0}{ }^{\prime}\right] \text {. }
$$

We require that (8) which automatically is satisfied for every quadratic shall hold for $y=x^{3}$. But this then determines $p=2 / 3$ or

$$
y_{1}=y_{0}+\frac{h}{4}\left(3 y_{2 / 3}^{\prime}+y_{0}^{\prime}\right) \text {. }
$$

In another connection this may be recognized as HeUN's formula used with $y_{-2}^{\prime}, y_{0}{ }^{\prime}, y_{-2}$ and $y_{1}$, respectively, replacing $y_{0}{ }^{\prime}, y_{2 / 3}^{\prime}, y_{0}$, and $y_{1}$. Now, however, we make use of our quadratic function $u(x)$. We cannot use (9) since $y_{2 / 3}^{\prime}$ is not known but from (7) with $x=x_{p}, p=2 / 3$ we find, letting $u\left(x_{p}\right)=y_{p}$,

$$
y_{2 / 3}=y_{0}+\frac{h}{3}\left(y_{2 / 3}^{\prime}+y_{0}^{\prime}\right)
$$

which provides the same algorithm type as (1) for finding $y^{\prime}{ }_{2 / 3}$ which is then substituted in (9) to obtain $y_{1}$. If (9) is considered as an integration formula it has an error term $\frac{h^{4} y^{\mathrm{IV}}}{216}$ assuming $y(x)$ has a continuous fourth derivative. The method is not symmetrical but it has been easy to calculate and more accurate than (1) in some calculations.

The application of the Gauss formula results if we choose the quadratic function

$$
u(x)=y_{0}+a_{1}\left(x-x_{0}\right)+a_{2}\left(x-x_{0}\right)^{2}
$$

subject to conditions: $x_{p}=x_{0}+p h, x_{q}=x_{0}+q h$,

$$
\begin{aligned}
& u_{p}{ }^{\prime}=f\left(x_{p}, u_{p}\right), \\
& u_{q}{ }^{\prime}=f\left(x_{q}, u_{q}\right),
\end{aligned}
$$

from which we obtain the function $u(x)$, with $u_{p}{ }^{\prime}=y_{p}{ }^{\prime}, u_{q}{ }^{\prime}=y_{q}{ }^{\prime}$ :

$$
u(x)=y_{0}+\frac{q y_{p}{ }^{\prime}-p y_{q}{ }^{\prime}}{q-p}\left(x-x_{0}\right)+\frac{y_{q}{ }^{\prime}-y_{p}{ }^{\prime}}{2(q-p) h}\left(x-x_{0}\right)^{2} .
$$

Again if we take $x=x_{1}$ in (13) we find with $y_{1}=u\left(x_{1}\right)$

$$
y_{1}=y_{0}+\frac{h}{2(q-p)}\left[(2 q-1) y_{p}^{\prime}+(1-2 p) y_{q}^{\prime}\right] \text {. }
$$

Now we require (14) which holds for quadratic functions to hold also for $y=x^{3}$ and $y=x^{4}$. As a result we obtain $p=1 / 2-1 / \sqrt{12}$ and $q=1 / 2+1 / \sqrt{12}$ (these are the zeros of the Legendre polynomial of degree 2 on $[0,1])$. Using the letters 
rather than the numbers when convenient we find from (13) and (14)

$$
\begin{aligned}
& y_{p}=y_{0}+\frac{p h}{2(q-p)}\left[(2 q-p) y_{p}{ }^{\prime}-p y_{q}{ }^{\prime}\right] \\
& y_{q}=y_{0}+\frac{q h}{2(p-q)}\left[(2 p-q) y_{q}{ }^{\prime}-q y_{p}{ }^{\prime}\right] \\
& y_{1}=y_{0}+\frac{h}{2}\left(y_{p}{ }^{\prime}+y_{q}{ }^{\prime}\right) .
\end{aligned}
$$

Hence, we use (15) and (16) to find by compound iteration $y_{p}{ }^{\prime}$ and $y_{q}{ }^{\prime}$ and then substitute in (17) to obtain $y_{1}$. The iteration procedure given by (15) and (16) converges provided $h<(\sqrt{3}+1) / m$ where $m=\max \left|\frac{\partial f}{\partial y}\right|$. Note that, as integration formulas, (9) and (17) are both trapezoidal but that the evaluation points do not coincide with the endpoints of the interval. The error in (17) as an integration formula may be written $\frac{h^{5} y^{(5)}}{4320}$.

The last method in continuation gives a picture of parabolic arcs joined continuously but not smoothly, each of which agrees in direction with that indicated by the differential equation at two points which are symmetrically placed in the interval. Thus this method is symmetrical or reversible theoretically, while that of equations (9) and (10) is not symmetrical.

Of the three trapezoidal methods which is best? The comparative study of methods for merit depends on so many features that the most one can hope for now is a qualitative judgment. The first method is simpler but not much more so than the second. If the symmetry is no consideration then one will expect the second method to be more accurate. The third method, on the other hand, is appreciably more difficult to use than the other two, although it is the most accurate and we believe it is also the most stable of the three methods. For automatic computing machines the use of irrational abscissas is no worse than use of, say, 1/3. We have tested these methods at the University of Wisconsin Numerical Analysis Laboratory and have found that for a linear differential equation the third method was significantly the most economical for a given accuracy. If roundoff error is likely to be bothersome, the presumably many fewer total number of steps required for the last method would minimize the difficulty. For general use on simple problems the second method should be better than the first.

We have neglected to give methods for obtaining starting values for the iteration procedure. We have done so, since it seems that we can use (10) with $y_{2 / 3}^{\prime}=y_{0}{ }^{\prime}$ for the second method, and $y_{0}+p h y_{0}{ }^{\prime}$ and $y_{0}+q h y_{0}{ }^{\prime}$ as first estimates of $y_{p}$ and $y_{q}$ for (15) and (16). The customary methods of guessing these values would make it more difficult to change $h$ repeatedly, which these procedures may claim as an advantage. The high speed computing machines make it possible to enjoy the advantages of intricate methods. They may make it virtually necessary on difficult problems to use methods of increasing complexity in order to minimize errors. 
We will present later a geometrical view of certain classical methods, and other new methods, some of which are non-polynomial in character. This presentation is made to indicate the value of the general approach we have made. The parabolic methods are special cases of what we have termed the parametric methods which include certain methods of Adams, Milne, Moulton, Obrechkoff, Ritz, and Galerkin, for example. Extension of results of this paper to higher order integration formulas is straightforward but would serve no useful purpose here as an illustration of our new point of view.

The Advanced Numerical Analysis class of Mr. Hammer has recently carried out rather extensive C.P.C. calculations to compare several methods for numerical solution of the differential equation $y^{\prime}=x^{2}+y^{2}$ with initial point $(0,1)$. For this problem the third method here was superior to the simple trapezoidal method. It is intended to publish these calculations separately. Mr. Orville Marlowe carried out calculations on simple linear differential equations including $y^{\prime}=y$ with initial point $(0,1)$ and concluded that here the third method was most economical for a given accuracy, partly due to the fact that no iteration is needed for linear equations.

University of Wisconsin

Preston C. Hammer

JACK W. HollingsWORTH

Madison, Wisconsin

General Electric Corporation

Schenectady, New York

Calculations in the University of Wisconsin Numerical Analysis Laboratory concerning methods in this report were financed by funds of the Wisconsin Alumni Research Foundation.

${ }^{1}$ W. E. Milne, Numerical Solution of Differential Equations, John Wiley and Sons, New York, 1953.

\section{Solving Systems of Linear Equations with a Positive Definite, Symmetric, but possibly Ill-conditioned Matrix}

Introduction. Often a system of linear equations to be solved has a matrix which is known in advance to be positive definite and symmetric. The normal equations for least squares fitting of a polynomial form such an example. However, if the polynomial is of reasonably high degree, the matrix of the normal equations is apt to be ill-conditioned. This may be seen by observing the origin of such matrices. In general they are of the following form:

$$
\begin{aligned}
& \sum_{i} x_{i}{ }^{0} \cdots \sum_{i} x_{i}{ }^{N-1} \sum_{i} x_{i}{ }^{N} \\
& \sum_{i} x_{i}{ }^{N} \cdots \sum_{i} x_{i}{ }^{2 N-1} \sum_{i} x_{i}{ }^{2 N} .
\end{aligned}
$$

Here the superscripts are exponents, $N$ is the degree of the polynomial, and the $x_{i}$ are the values of the argument at which the data is given. For sums of high 